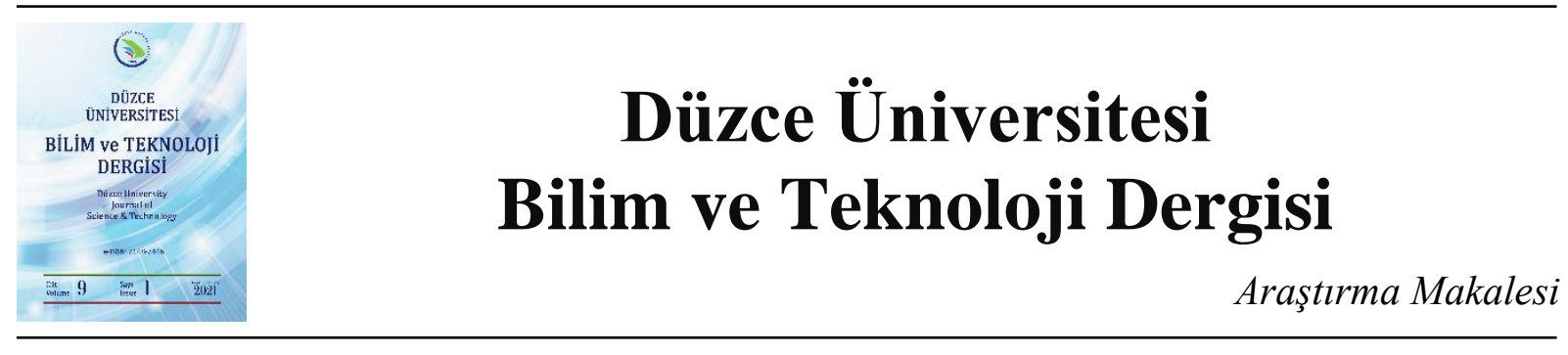

\title{
4-propoxy-biphenyl-4-carbonitrile Nematik Sivı Kristalinin Dielektrik Özellikleri
}

\author{
D Pınar Özden a, (D) Atilla Eren Mamuk ${ }^{\mathrm{a}}$, (D) Şadan Özden a, (iD Nejmettin Avca a * \\ ${ }^{a}$ Fizik Bölümü, Fen Fakültesi, Muğla Sıtkı Koçman Üniversitesi, Muğla, TÜRKIYYE \\ * Sorumlu yazarın e-posta adresi: navci@mu.edu.tr
}

DOI : 10.29130/dubited.791920

\begin{abstract}
ÖZET
$\mathrm{Bu}$ çalışmada, nematik fazdaki 4-propoxy-biphenyl-4-carbonitrile'in sıcaklığa bağlı dielektrik geçirgenliğinin ölçümleri raporlanmıştır. Bu amaçla $1 \mathrm{kHz}$ ila $1 \mathrm{MHz}$ frekans aralığında sıcaklığa bağlı dielektrik karakterizasyonu gerçekleştirilmiştir. Sözü edilen malzeme nematik fazda pozitif dielektrik anizotropik özellik göstermektedir. Ayrıca uzun eksen etrafında molekül rotasyonuna karşılık gelen dielektrik gevşeme frekansları sıcaklığın bir fonksiyonu olarak ölçülmüş ve gevşeme sürecinin aktivasyon enerjisi Arrhenius metodu vasıtasıyla belirlenmiştir.
\end{abstract}

Anahtar Kelimeler: Sıvı kristal, nematik, dielektrik geçirgenlik, aktivasyon enerjisi

\section{The Dielectric Properties of 4-propoxy-biphenyl-4-carbonitrile Nematic Liquid Crystal}

\begin{abstract}
In the present investigation, the temperature dependent measurements of dielectric permittivity for 4-propoxybiphenyl-4-carbonitrile was reported in the nematic region. Temperature-dependent dielectric characterization was carried out over the frequency range of $1 \mathrm{kHz}$ to $1 \mathrm{MHz}$. The compound shows positive dielectric anisotropy in the nematic phase. The dielectric relaxation frequencies corresponding to the molecular rotation around the long axis were measured as a function of temperature. The activation energy of relaxation process was determined by means of the Arrhenius relation.
\end{abstract}

Keywords: Liquid crystal, nematic, dielectric permittivity, activation energy 


\section{GIRIS}

Sıvı kristallerin dielektrik davranışı, mezojenlerin toplu tepkisinin yanı sıra, moleküler özellikleri de yansıtmaktadır. Makroskobik polarizasyon ve iç elektrik alan vasıtasıyla moleküler tepki arasında bir bağlantı vardır. Dolayısıyla, sıvı kristal fazların dielektrik özelliklerinin moleküler düzeyde tanımlanması için anizotropik ortam içinde iç elektrik alan koşullarının belirlenmesi gerekmektedir. $\mathrm{Bu}$ koşulların belirlenmesi genellikle oldukça zordur. $\mathrm{Bu}$ nedenden dolayı sıvı kristallerin dielektrik özelliklerinin incelenmesi oldukça önemlidir [1-5].

Sıvı kristal malzemeler günlük yaşamda kendilerine geniş uygulama alanları bulmaktadırlar. Bu ilgi çeken malzemelerin güçlü uygulamalarının geniş yelpazesi akademik ve endüstriyel araştırmalarda yeni çalışma alanları açmaktadır. Sıvı kristaller hem katı kristallerin hem de izotropik sıvıların özelliklerini sergileyen özel malzemelerdir. Böylece sıvı kristaller benzersiz elektriksel ve optik özelliklere sahiplerdir. İncelenmekte olunan molekülün şekline ve kimyasal özelliklerine bağlı olarak çeşitli sıvı kristal fazların sergilenmesi mümkündür. Materyalde görülen bu sıvı kristalik fazların her biri mezofaz olarak adlandırılmaktadır. Bu sınıflandırma belirli sıvı kristal fazdaki moleküllerin düzenlenmesi ve molekül dizilimine dayanmaktadır. Nematik sıvı kristaller uzun erimli yönelimsel düzene sahiplerdir. Ancak öteleme düzenleri yoktur. Nematik sıvı kristallerdeki tüm moleküllerin ortalama yönelimi yön vektörü olarak tanımlanmaktadır. Nematik sıvı kristal mezofaz, en basit dielektrik davranışa sahiptir ve nematikler için elde edilen kalitatif sonuçlar diğer mezofazlardaki molekül dinamiklerinin anlaşılması için de avantaj sağlamaktadır. Nematik sıvı kristal mezofazın en etkin özelliği, küçük değerlerdeki elektrik alanlara yanıt vermesidir ve bu durum söz konusu sıvı kristalleri elektro-optik cihazlarda ilginç bir malzeme haline getirmektedir.

4-alkoxy-4-cyanobiphenyls (mOCBs) uzun zincirli alifatik bileşikler sınıfina ait bir sıvı kristal gurubudur. Renksiz, kimyasal ve fotokimyasal olarak nispeten kararlı, düşük erime noktasına sahip sıvı kristaller olmaları nedeniyle endüstride yaygın olarak kullanılmaktadırlar [6-34]. Terminallerinde siyano (cyano-CN) ve alkoksil (alkoxyl) grupları olan polarize edilebilir aromatik halkalara sahiptirler $[7,11,14,15,27]$.

Son zamanlarda, esnek bir ayırıcı ile birleştirilen iki mezojenik üniteden oluşan bazı dimerik moleküller, standart nematik fazdan farklı olarak genellikle yeni tip bir nematik fazı sergilemektedirler. Bu tür molekül yapısına sahip mezofazlar burulu-bükümlü (twist-bend) nematik fazı (Ntb) olarak adlandırılırlar. Bu yeni fazda moleküllerin kendileri etkin bir şekilde kiral olmamalarına rağmen yön vektörü dizilimi helikoidal (helicoidal) bir yapıdır [35-39]. Yön vektörü sarmal eksene göre eğilir ve kendiliğinden meydana gelen adım uzunluğu tipik olarak $10 \mathrm{~nm}$ civarındadır [35]. Yaygın olarak çalışılan CB6OCB molekülü bu tür sıvı kristal dimerlere bir örnektir [36]. 4-propoxy-biphenyl-4carbonitrile sıvı kristalinin aromatik ve alifatik kısımları, bu dimerin yarısına hemen hemen eşittir. 4propoxy-biphenyl-4-carbonitrile molekülünün dielektrik özelliklerin incelenmesi $\mathrm{Ntb}$ fazı sergileyen malzemeler hakkında bilgi verecektir. Bu çalışmada ilgili nematik malzemelerin daha iyi anlaşılabilmesi için 4-propoxy-biphenyl-4-carbonitrile üzerinde dielektrik geçirgenliğinin frekans ve sıcaklığa bağımlılı̆g incelenmiştir.

\section{MATERYAL VE YÖNTEM}

Bu çalışmada incelenen nematik sıvı kristal 4-propoxy-biphenyl-4-carbonitrile'dir ve BDH şirketinden temin edilmiştir. Malzemenin faz geçiş sıcaklıkları ve tekstürleri Olympus BX-51P marka polarizasyon mikroskobu ve Linkam marka sıcaklık kontrollü 1sitıcı ünitesi kullanılarak belirlenmiştir. Malzeme soğutma esnasında yaklaşık olarak $68.1^{\circ} \mathrm{C}$ ile $56.9^{\circ} \mathrm{C}$ sıcaklık değerleri arasında monotropik nematik fazı sergilemiştir. Isıtma esnasında ise izotropik faza $74.2{ }^{\circ} \mathrm{C}$ sıcaklığında geçmiştir. Molekülün kimyasal yapısı ve izotropik sıvı nematik faz geçişini gösteren tekstürü Şekil 1'de verilmektedir. 


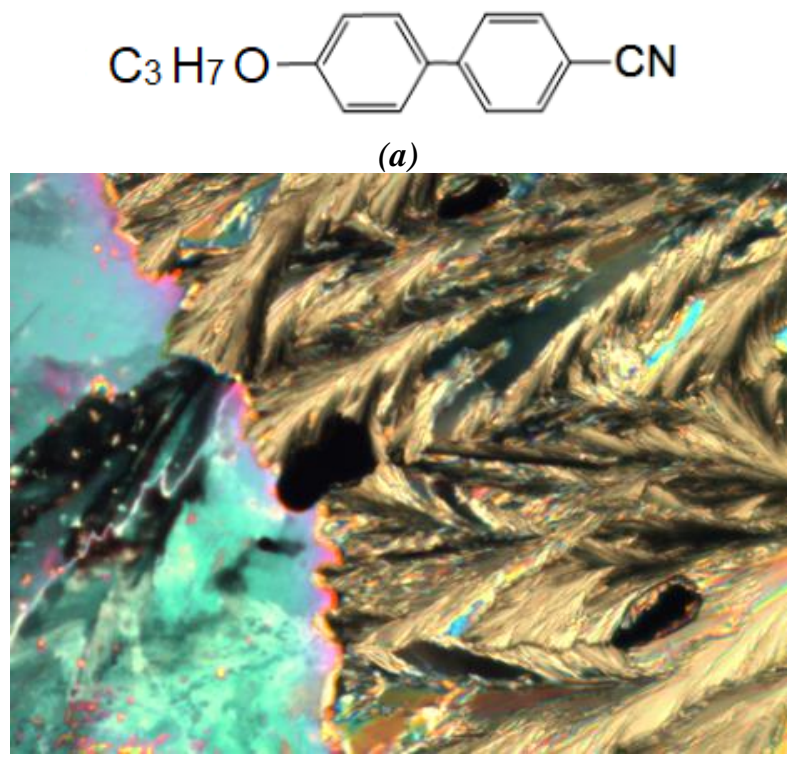

(b)

Şekil 1. (a) 4-propoxy-biphenyl-4-carbonitrile'nin kimyasal yapısı ve (b) $74{ }^{\circ} \mathrm{C}$ 'deki faz geçiş tekstürü.

Dielektrik ölçümler için sandviç hücreler belirtilen aşamalarda hazırlanmıştır: İlk olarak, indiyum kalay oksit (ITO) tabakalı şeffaf cam tabakalar, deterjanlı su çözeltisi ile dolu bir ultrasonik banyoda 30 dakika boyunca yıkanmışlardır. Daha sonra, cam tabakalar deiyonize su ile durulanmış ve izopropanol ile yıkanmışlardır. Yıkanan cam tabakalar metanol ve etanol ile birkaç kez temizlenmiş ve $60^{\circ} \mathrm{C}$ sıcaklıkta kurutulmuşlardır. Kurutulmuş yüzeyler organik kirliliğin ortadan kaldırılması için beş dakika UV ışığa maruz bırakılmışlardır. Fotolitografi yöntemi kullanarak, alanı $25 \mathrm{~mm}^{2}$ olan kare şeklinde ITO elektrotlar oluşturulmuştur.

Bu çalışmada, cam hücrelerde sıvı kristal moleküllerini farklı şekillerde yönlendirebilmek için cam yüzeyleri farklı türde polimerler ile kaplanmıştır. Polimer kullanarak cam hücrelerde yüzeylerin işlenmesi düzgün yönlendirilmiş sıvı kristal hücre üretimi için kullanılan en yaygın yöntemdir. Çünkü bu tekniğin uygulanması diğer yönlendirme metotlarına nazaran kolaydır. Bu yöntemle, sıvı kristal molekülleri için düzlemsel yönelim elde edilebilmektedir. LCD (sıvı kristal ekran - Liquid Crystals Display) hücrelerinde sıvı kristalin yönelimi için bu teknik kullanılarak mükemmel elektro-optik performans elde etmek mümkün olmaktadır. Cam yüzeylerde planar yönelim elde etmek için PI2555 (HD Microsystems) ve T9039 (HD Microsystems) poliimidler kullanılarak 1/4 oranında çözelti hazırlanmış ve $300 \mathrm{rpm}$ hızındaki döndürmeli kaplama cihazıyla çözelti homojen bir şekilde cam yüzeyinde dağıtılmıştır. Kaplanan cam yüzey üzerinde $10 \mathrm{~nm}$ 'lik ince bir film oluşturulmuştur. $\mathrm{Bu}$ filmin kalınlığı hazırlanan çözeltinin oranına göre değişmektedir. Daha sonra bir dakika süreyle $90^{\circ} \mathrm{C}$ 'de yumuşak ısıl işlem yapılmış ve buna ek olarak 1 saat süre ile $275^{\circ} \mathrm{C}^{\prime}$ de sert 1 șıl işlem yapılmıştır. Daha sonra poliimid kaplı cam yüzeyler 800-850 Pa basınca sahip bir blok üzerine sarılı temiz bir kadife kumaş kullanılarak belirlenen tek bir yön boyunca en az 10 defa sürtülerek işlenmiştir. İşlenmiş camlar, cam boncuklar içeren ve UV-ış1k altında polimerize olan Norland yapışkan (NOA-65) kullanılarak sürtülme yönüne zıt yönde birleştirilmiş ve sonuçta planar yönelime sahip sandviç hücreler elde edilmiştir.

Homeotropik yönelim için ise SI1211 (HD Microsystems) ve T9039 (HD Microsystems) poliimidler kullanılarak 1/3 oranında çözelti hazırlanmış ve $300 \mathrm{rpm}$ hızındaki döndürmeli kaplama cihazıyla çözelti homojen bir şekilde cam yüzeyinde dağıtılmıştır. Kaplanan cam yüzey üzerinde $15 \mathrm{~nm}$ 'lik ince bir film meydana gelmesi sağlanmıştır. Döndürmeli kaplama tekniği ve bu çözelti kullanılarak cam yüzeyinde ince bir homeotropik tabaka oluşturulmuştur. Daha sonra bir dakika süreyle $90^{\circ} \mathrm{C}$ 'de yumuşak 1 sıl işlem yapılmış ve buna ek olarak 1 saat süre ile $180^{\circ} \mathrm{C}$ 'de sert 1 sıl işlem yapılmıştır. Hazırlanan yönelmiş durumdaki sandviç cam hücreler, sıvı kristal malzemelerin izotropik sıv1 sıcaklığına kadar 1sıtılmış̧ır. Bu sıcaklıkta sıvı kristal malzemeler kapilyar etki sayesinde cam hücre içerisine doldurulmuştur. Örnek 
hücreler istenilen sıcaklığa kadar yavaş bir şekilde soğutularak moleküllerin düzenli bir yönelim elde etmeleri sağlanmıştır. Daha sonra malzemelerin buharlaşmasını veya cam hücreden dışarı akmasını önlemek amacıyla diğer uçlar da epoksi tutkal ile yapıştırılmıştır. Deneyler için kullanılan tipik hücre kalınlığı yaklaşık $8 \mu \mathrm{m}$ 'dir. Sıv1 kristal ile doldurulmuş hücreler Linkam LTS350 1sitıcı tarafından 1sıtılmış ve sıcaklık $\pm 0.1^{\circ} \mathrm{C}$ hassasiyete sahip olan TMS94 (Linkam Scientific Instruments) sicaklık kontrolcüsü ile kontrol edilmiştir.

Sıvı kristallerin dielektrik yanıtının sıcaklığa bağlılığı, elektrik alanın varlığında polar moleküllerin hem kolektif hem de kolektif olmayan süreçlerinin detayları ve dinamikleri hakkında değerli bilgileri sağlamaktadır. Bununla birlikte, yönelimsel düzen parametresi sıcaklığa bağlıdır, moleküllerin dipolleri ve düzen parametresi konformasyonel dağılımdan etkilenmektedir [1-6]. Sıv1 kristal bir malzemenin dielektrik özellikleri hem frekans $(f)$ hem de sicaklığa $(T)$ bağlıdır [34-40]. Dielektrik geçirgenlik $\varepsilon *$ $(f, T)=\varepsilon^{\prime}(f, T)-i \varepsilon^{\prime \prime}(f, T)$ şeklindedir. Burada $\varepsilon^{\prime}(f, T)$ ve $\varepsilon^{\prime \prime}(f, T)$ sirasiyla dielektrik geçirgenliğinin gerçel ve sanal kısımlarıdır. Dielektrik karakterizasyon için $1 \mathrm{kHz}$ den $1 \mathrm{MHz}$ ’e kadar değişik frekanslarda " $a c$ " gerilim uygulayabilen Schlumberger SI1260 marka cihaz kullanılmıştır. Planar ve homeotropik yönelmiş hücreler sıvı kristal ile doldurulmadan önce boş hücrelerin kapasitansı ölçülmüştür. Daha sonra istenilen sıcaklıklarda dolu hücrelerin kapasitans verileri alınmıştır. Moleküllerin yeniden yönlenmesini ve doğrusal olmayan etkileri önlemek için $0.2 \mathrm{~V}$ gibi oldukça düşük bir ac gerilim uygulanmıştır. Tüm ölçümlerde sıcaklık, katı kristal faza girmeden adım adım düşürülmüştür.

\section{SONUCLAR}

4-propoxy-biphenyl-4-carbonitrile sıvı kristal molekülü, iki ana polar $\mathrm{C}-\mathrm{O}$ ve - $\mathrm{CN}$ gruba ayrılmaktadır. Bu iki grup, elde edilen toplam kalıcı dipol momente katkıda bulunmaktadır ve dipol momentin yönü moleküllerin uzun ekseni (boyuna) ile tam olarak aynı yönde değildir. Alkoksil zinciri muhtemelen nematik fazda genişletilmiş bir konfigürasyondadır. Çünkü moleküllerin uzun ekseni, iki benzen halkasının merkezlerini birleştiren doğrultuya eğilmesi gerekmektedir [11,27,28]. Böylece, dipol momentinin boyuna $\left(\mu_{l}\right)$ ve enine $\left(\mu_{t}\right)$ bileşenleri ile ilintili en az iki dağılım bölgesi dielektrik spektrumda meydana gelmektedir [1-5]. Homeotropik ve planar yönelimli 4-propoxy-biphenyl-4-carbonitrile sıvı kristalin dielektriksel geçirgenliğinin sıcaklığa göre değişimleri Şekil 2'de gösterilmiştir. İzotropik fazdaki dielektrik geçirgenlik, planar ve homeotropik yönelimli hücreler için hemen hemen aynıdır. Sıcaklık $67{ }^{\circ} \mathrm{C}$ 'ye geldiğinde, dielektrik geçirgenliğin iki farklı yola ayrıldığ 1 ve $2 \mathrm{kHz}$ ile $20 \mathrm{kHz}$ arasında neredeyse sabit kaldığı görülmektedir. Sıvı kristal moleküllerin elektrik alan salınımları sırasında termal denge durumuna ulaşmak için yeterli zamana sahip oldukları ve indüklenen dipol momentin uygulanan elektrik alanla aynı fazda tepki verdiği söylenebilir [35]. Ancak $\varepsilon^{\prime}(f, T) 100 \mathrm{kHz}$ ile $1 \mathrm{MHz}$ frekans aralığında keskin bir şekilde azalmaktadır. Çünkü sıvı kristal moleküllerinin, elektrik alan salınımları sırasında termal denge durumuna ulaşmak için yeterli zamanları yoktur ve moleküller, uzun eksenleri etrafında dönme için net dipol momenti sağlayamazlar. Belirli sıcaklıklarda ve yüksek bir frekans aralığında $(20 \mathrm{kHz}$ ila $400 \mathrm{kHz})$ frekansın artması ile $\varepsilon^{\prime \prime}(f, T)$ 'nın büyüklüğü artar. $\varepsilon^{\prime \prime}(f, T)$ 'nın $10 \mathrm{kHz}$ 'in altındaki düşük frekanslarda artması, safsızlık ile iyonik iletkenliğin baskınlığına bağlanabilir. Nematik fazda dielektrik geçirgenliğin paralel bileşeni dik bileşeninden daha büyüktür. Çünkü dipol momentin paralel bileşeninin dielektrik geçirgenliğe katkısı dik bileşenden daha büyüktür [1-5]. Nematik fazda moleküllerin hareketleri esas olarak moleküllerin uzun ekseni etrafında dönmesidir. Maksimum kayıp (loss) frekansı, dipollerin gevşeme frekansları ile ilgilidir [1,3,40]. Gevşeme frekansı, nematik moleküllerin kısa ekseni boyunca yön değiştirmesi nedeniyle homeotropik hizalanmış moleküller için daha yüksek frekansa doğru kayar ve sıcaklık azaldıkça azalır. Arrhenius davranışından beklendiği gibi difüzyon sabitinin sıcaklığa bağımlılığı nedeniyle sıcaklığın azalması ile gevşeme frekansı genel bir artırma eğilimi göstermektedir. 


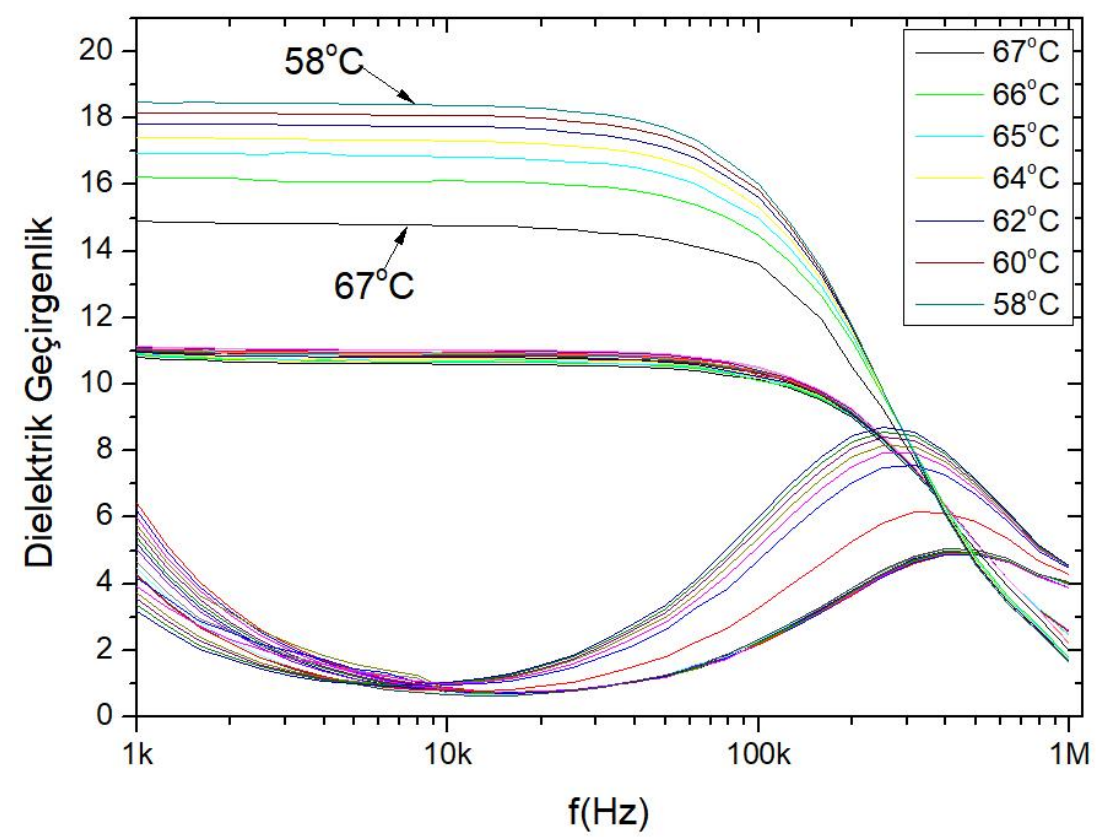

(a)

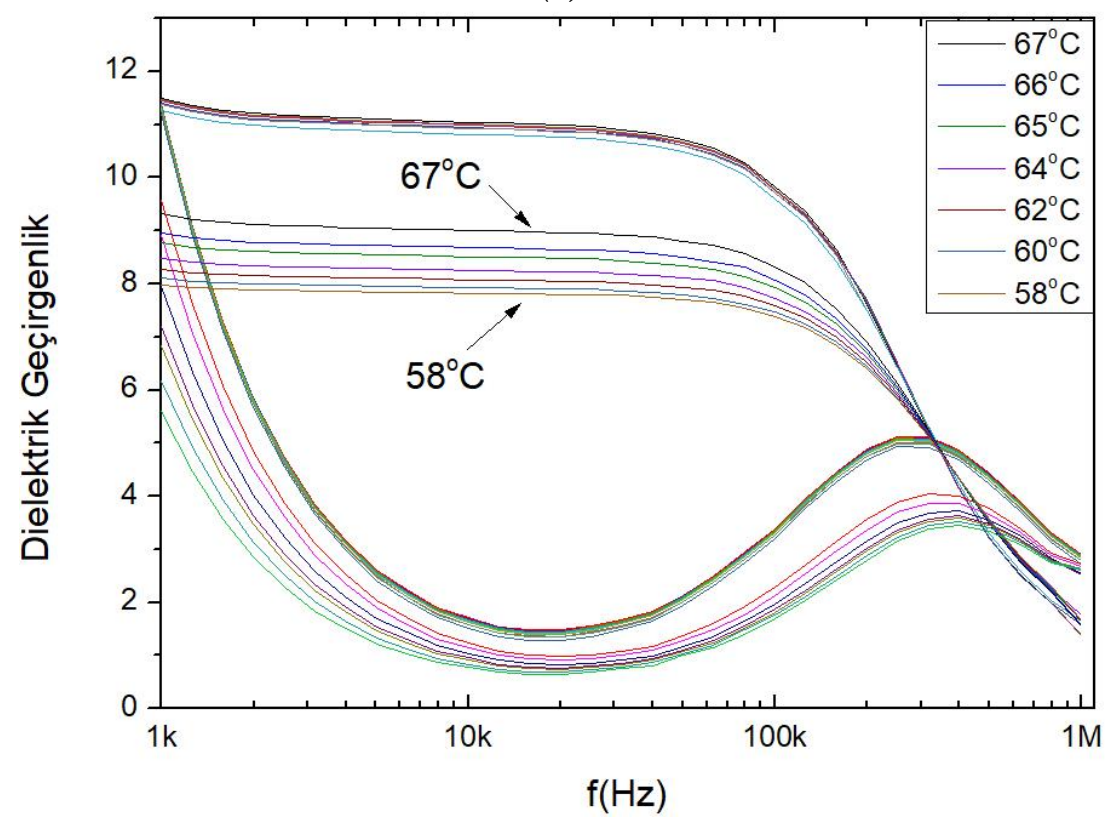

(b)

Şekil 2. 4-propoxy-biphenyl-4-carbonitrile sıvı kristalin dielektrik geçirgenliğinin frekansa göre değişimi (a) homeotropik yönelim ve (b) planar yönelim.

Şekil 3'teki frekansa bağlı dielektrik spektrumlarından sadece güçlü bir soğurum süreci gözlenmektedir. Gevşeme frekansı ve simetrik dağılım parametresini elde etmek için dielektrik spektrumları aşağıda verilen değiştirilmiş Cole-Cole kompleks dielektrik geçirgenlik fonksiyonuna uydurulmuştur (fitting) [40].

$$
\begin{aligned}
& \varepsilon *=\varepsilon^{\prime}-i \varepsilon^{\prime \prime}=\varepsilon_{\infty}+\frac{\delta \varepsilon^{a}}{1+\left(i \omega \tau_{r}\right)^{1-\alpha}}-i \frac{\sigma}{\omega \varepsilon_{o}} \quad(a=/ /, \perp) \\
& \varepsilon^{\prime}=\varepsilon_{\infty}+\delta \varepsilon^{a} \frac{1+\left(\omega \tau_{r}\right)^{1-\alpha} \sin \left(\frac{\pi \alpha}{2}\right)}{1+2\left(\omega \tau_{r}\right)^{(1-\alpha)} \sin \left(\frac{\pi \alpha}{2}\right)+\left(\omega \tau_{r}\right)^{2(1-\alpha)}}
\end{aligned}
$$




$$
\varepsilon^{\prime \prime}=\delta \varepsilon^{a} \frac{\left(\omega \tau_{r}\right)^{1-\alpha} \cos \left(\frac{\pi \alpha}{2}\right)}{1+2\left(\omega \tau_{r}\right)^{(1-\alpha)} \sin \left(\frac{\pi \alpha}{2}\right)+\left(\omega \tau_{r}\right)^{2(1-\alpha)}}+\frac{\sigma_{D C}}{\omega \varepsilon_{o}}
$$

Burada, $\omega$ açısal frekansıdır $(\omega=2 \pi f), \sigma_{D C}$ DC iletkenlik, $\varepsilon_{o}$ vakumdaki dielektrik sabitidir. $\alpha$ gevşeme sürelerinin simetrik dağılımından sorumlu bir parametredir (Cole - Cole dağıtım parametresi), $\delta \varepsilon^{a}=\left(\varepsilon_{s}-\varepsilon_{\infty}\right)$ gevşeme sürecine (veya dielektrik artışa) bağl1 dielektrik güçtür. $\tau_{r}$ gevşeme zamanıdır $\left(\tau_{r}=1 / 2 \pi f_{r}\right) . \delta \varepsilon$, toplam dipol moment $(\mu)$ ile orantılıdır. $f_{r}$ gevşeme frekansıdır. $\varepsilon_{\infty}$ optik daldaki dielektrik geçirgenliğidir. $\varepsilon_{s}$ düşük frekanstaki dielektrik geçirgenliğidir. $\alpha$ 'nın değerleri ColeCole grafiklerinden elde edilmiş ve 0.01 olarak bulunmuştur. Gevşeme süreci neredeyse Debye tipi bir davranış olduğunu göstermektedir. Bu nedenle, moleküllerin uzun ekseni etrafındaki dipolar rotasyona bağlı gevşeme süreci davranışı da neredeyse Debye tipindedir. Artan sıcaklık ile dielektrik gücündeki artış, gevşeme frekansının dağılım simetrisindeki artışından kaynaklanmaktadır. Örnek için uydurma işlemi sonucu DC iletkenliği $\left(\sigma_{D C}\right) 1.11 \times 10^{-13} \mathrm{~S} / \mathrm{m}$ olarak bulunmuştur.

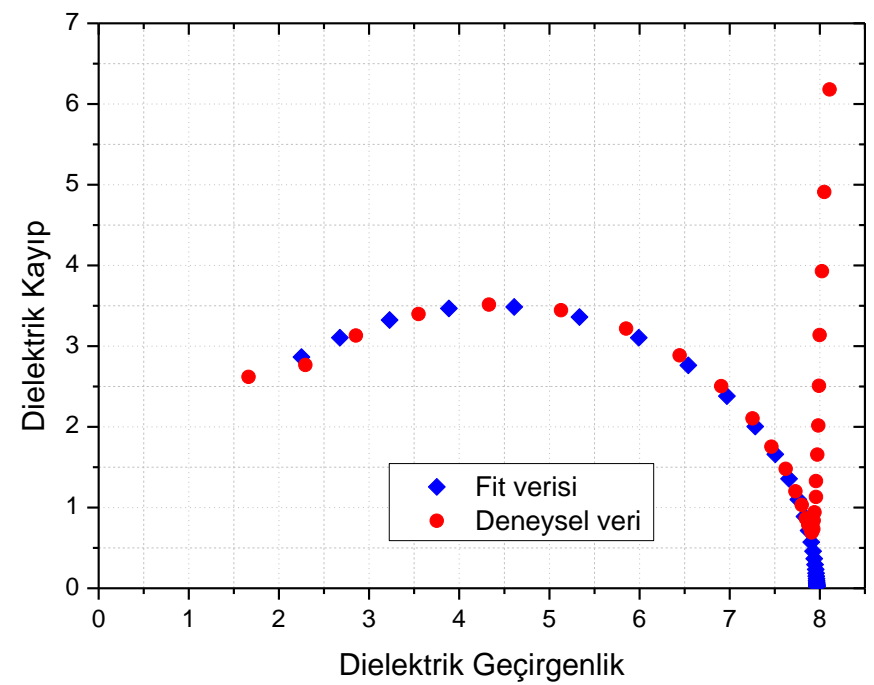

(a)

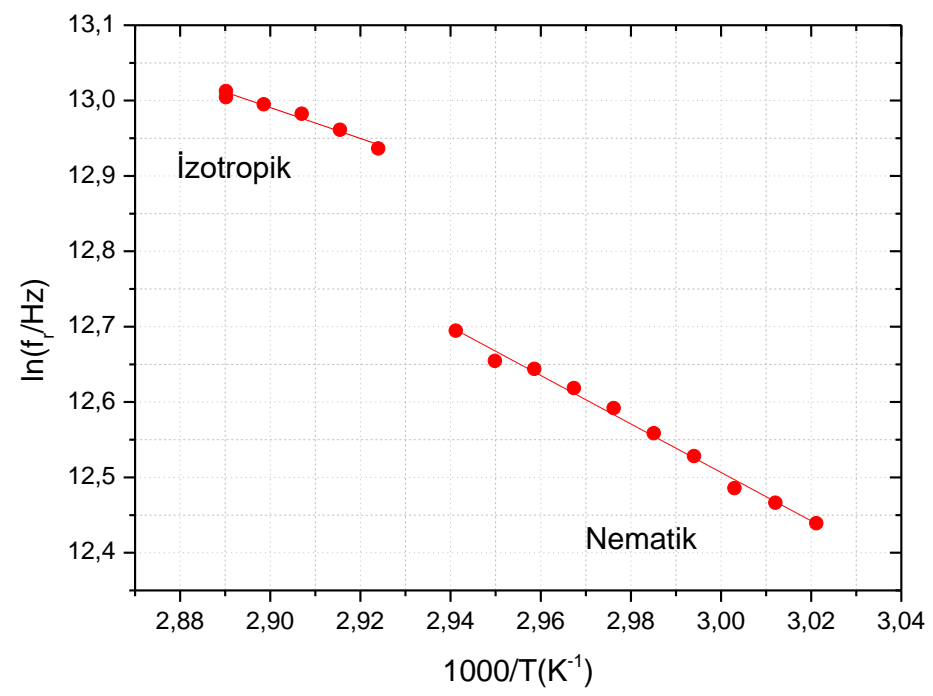

(b)

Şekil 3. (a) 4-propoxy-biphenyl-4-carbonitrile'nin Cole-Cole ĕgrisi $\left(58^{\circ} \mathrm{C}\right)$ ve (b) eşik frekansların sıcaklı̆̆ının tersine göre değişimi.

Bu çalışmada, dielektrik geçirgenliğinin hem gerçel hem de sanal kısımları analiz edilmiştir. Deneysel dielektrik spektrumun Cole-Cole eğrilerine uydurulması, en küçük kareler metodu ile yapılmıştır. ColeCole eğrilerinin merkezleri gerçel eksene değdiğinde $\left(\varepsilon^{\prime \prime}=0\right)$, sadece bir gevşeme frekansı vardır [40]. 
Dielektrik sabitlerinin gerçel ve sanal kısımları Kramers-Kronig mekanizması ile ilişkilidir. Planar yönelimli hücrenin $58^{\circ} \mathrm{C}$ 'deki Cole -Cole eğrisi Şekil 3a'da gösterilmiştir ve dielektrik fonksiyonun tek bir gevşeme süresi ile tanımlanabileceğini göstermektedir. Sıcaklık artışı ile merkez dairenin yarıçapı artar ve dielektrik geçirgenliğin gerçel kısımları daha yüksek değerlere kayar. Burada eğrinin yarıçapı en küçük kareler çemberine uydurulmuş ve böylelikle gevşeme frekansına karşl1ık gelen frekans değerleri belirlenmiştir.

Şekil 3b'de görüldüğü gibi gevşeme frekansı $\left(f_{R}\right)$ sıcaklıkla artmaktadır. Sıcaklıkla uyarılan bu süreç dielektrik gevşemenin aktivasyon enerjisi ile açiklanabilir. Arrhenius denklemi $[1,5]$

$f_{R}=\operatorname{Aexp}\left(\frac{-W_{A}}{N_{A} k_{B} T}\right)$

şeklindedir. Burada $W_{A}, N_{A}$ ve $k_{B}$ sirasıla aktivasyon enerjisi, Avagadro sayısı ve Boltzmann sabitidir. $N_{A}=6.02205 \times 10^{23} \mathrm{~mol}^{-1}$ ve $k_{B}=1.38066 \times 10^{-23} \mathrm{~J} . \mathrm{K}^{-1}$ dir. Sicaklığın tersine karşı $\ln \left(f_{R}\right)^{\prime} \operatorname{nin}$ eğimi, en küçük kare uydurma yöntemi ile elde edilebilir. Nematik ve izotropik faz durumdaki aktivasyon enerjileri bu yöntemle belirlenmiştir. İzotropik fazdaki aktivasyon enerjisi $17.04 \mp 1.44$ $\mathrm{kJ} /$ moldur. Gevşeme frekansı viskozite ile ters orantılı olduğundan gevşeme frekanslarında sıcaklıkla azalması kaçınılmazdır [1-3]. $W_{A}$ 'nin nematik fazdaki değeri $26,74 \mp 0.08 \mathrm{~kJ} /$ moldur ve moleküllerin uzun ekseni etrafındaki takla (flip-flop) hareketi olağandır. Çünkü, moleküllerin kısa ekseni etrafındaki yeniden yönlendirilmesi genellikle $\mathrm{GHz}$ seviyesindeki frekanslarda gözlenir [40]. Nematik fazda nematik bariyer potansiyelinin varlığı nedeniyle, bu süreç güçlü bir şekilde engellenir ve bu nedenle izotropik fazdan önemli ölçüde daha düşük frekanslarda görülür [41,42]. Ölçümler nematik fazdaki her iki yön için de gerçekleştirildiğinde sadece moleküllerin uzun ekseni etrafındaki rotasyon hareketi önemli farklılıklar gösteren bir harekettir. Moleküllerin uzun ekseni etrafındaki yönlendirme frekans1 molekülün geometrik şekline, atalet momentine ve çevrenin etkisine bağlıdır [1-5].

Onsager teorisinin Maier ve Meier tarafından sıvı kristallere genişletilmiş hali [42]

$$
\begin{aligned}
& \varepsilon_{/ /}-1=\varepsilon_{o}{ }^{-1} N F h\left\{\bar{\alpha}+\frac{2}{3} \Delta \alpha S+F g_{/ /} \frac{\mu}{3 k T}\left[1-\left(1-3 \cos ^{2} \theta\right) S\right]\right\} \\
& \varepsilon_{\perp}-1=\varepsilon_{o}{ }^{-1} N F h\left\{\bar{\alpha}-\frac{1}{3} \Delta \alpha S+F g_{\perp} \frac{\mu}{3 k T}\left[1+\frac{\left(1-3 \cos ^{2} \theta\right)}{2} S\right]\right\} \\
& \Delta \varepsilon=\varepsilon_{o}{ }^{-1} N F h\left\{\bar{\alpha}-F \frac{\mu}{2 k T}\left(1-3 \cos ^{2} \theta\right)\right\}
\end{aligned}
$$

şeklindedir. Burada, $N$ moleküllerin sayı yoğunluğudur. $\Delta \alpha$ polarizasyonun anizotropisidir. $\bar{\alpha}$ ortalama polarizasyonudur. $\mu$ elde edilen dipol momentidir. $\theta$ dipol momentinin yönü ile moleküllerin uzun ekseni arasındaki açıdır. $k$ Boltzmann sabitidir. $F=\left(1-\frac{(\bar{\varepsilon}-1) 2 N \bar{\alpha}}{2(\bar{\varepsilon}+1) 3 \varepsilon_{o}}\right)^{-1}$ reaksiyon alan faktörüdür. $S$ düzen parametresidir. $g_{/ /, \perp}$ Fröhlich-Kirkwood korelasyon faktörüdür. $h=3 \bar{\varepsilon} /(2 \bar{\varepsilon}+1)$ boşluk alan faktörüdür.

Dielektrik geçirgenliğin gerçel kısmının hem homeotropik hem de planar yönelime sahip sıvı kristal için $2 \mathrm{kHz}$ ila $20 \mathrm{kHz}$ arasında neredeyse sabit kaldığı Şekil 2'de açıkça görülmektedir. Gerçel kısmın sabit kaldığ 1 bu aralıktan uygun bir frekans değeri seçilerek dielektrik sabitleri bulunmuştur. Bu bağlamda, 4-propoxy-biphenyl-4-carbonitrile sıvı kristalinin dielektrik sabitleri ( $\varepsilon_{/ /}$and $\left.\varepsilon_{\perp}\right) 2 \mathrm{kHz}$ 'de ölçülmüş ve sıcaklık değişimleri Şekil 4a'da gösterilmiştir. $\varepsilon_{/ /}$ve $\varepsilon_{\perp}$ sırasılyla dielektrik sabitinin moleküle paralel ve dik bileşenleridir. Sıcaklık izotropik - nematik faz geçişine indirildiğinde $\varepsilon_{/ /}$ve $\varepsilon_{\perp}$ davranışlarında birinci dereceden faz geçiş ile tutarlı bir süreksizlik mevcuttur. Maier-Meier modelinin önerdiği gibi sıcaklığın azalması ile $\varepsilon_{/ /}$monoton şekilde artarken [42] $\varepsilon_{\perp}$ ise tam tersi azalır (Şekil 3a). Oda sıcaklığında molekül eksenine paralel dielektrik sabitinin değeri yaklaşık 18 ve molekül eksenine dik 
dielektrik sabitinin değeri ise yaklaşı 8 dir. Çünkü moleküller oldukça güçlü eksensel dipol momente sahiptir.

Faz geçiş bölgesi civarındaki dielektrik geçirgenliğinin ortalama değerinin $\left[\varepsilon_{a v}=\left(\varepsilon_{/ /}+2 \varepsilon_{\perp}\right) / 3\right]$ izotropik bölgedeki değerinden daha düşük olduğu bulunmuştur. Bu da nematik fazdaki güçlü dipol momentleri ile moleküllerin antiparalel hizalanmasına atfedilebilir [35,40]. Gözlenen durum güçlü kutuplanmaya sahip sıvı kristal malzemelerin önceki araştırmacılar tarafından bildirilen teori ve deney sonuçlarıyla uyumludur $[31,32,40,43,44]$. Aksine bir örnek olarak, di-alkil azobenzen gibi polar olmayan moleküllerin uzun menzilli nematik düzenindeki bir azalma, kısa menzilli veya en yakın komşu moleküllerin antiparallel dizilimi oluşturmasından dolayı böyle bir süreksizlik göstermezler [45,46]. Sicaklığa göre nematik fazdaki dielektrik güç (strength) ve statik değişimler Şekil 4b'de verilmiştir. 2 kHz'de statik dielektrik geçirgenlik değeri, dielektrik geçirgenlik değerinden bir miktar yüksektir. Büyük dielektrik artış, $-\mathrm{CN}$ grubu tarafindan oluşturulan daha büyük boyuna dipol momentten kaynaklanır $[11,21,23,26-28]$. Fenil halkalar, $-\mathrm{CN}\left(\mu_{C N}=4.05 \mathrm{D}\right)$ ve $\mathrm{C}-\mathrm{O}\left(\mu_{C O}=0.8 \mathrm{D}\right)$ gruplar elektronik bulutunun oksijen atomundan azot atomuna geçişine neden olurlar. Böylelikle negatif yük azot atomuna indirgenir ve pozitif yük oksijen atomunun üzerine yerleşir. Bir molekülün -CN bağı, diğer molekülün $\mathrm{C}-\mathrm{O}$ bağının yakınında bulunur $[11,15,27,28]$. Alkoksi zinciri, diğer molekülün polar-CN grubundaki azottan önemli ölçüde daha fazla gerilmez. Her molekül, uzun ekseni boyunca önemli ölçüde yönlendirilen dipol momenti ile karakterize edilir [7,11,21,27]. Çünkü X-1şını kırınım çalışmaları -CN grubunun güçlü dipol momentleri nedeniyle çekirdek bölgelerin üst üste bindiğini göstermiştir [18,31,32]. Ek olarak, O-C, C-O ve C-C bağları nedeniyle moleküller sınırlı bir dönüşe sahiptir [23,30]. Çünkü alkil zincirinin fenilen halkasına olan etkisi, zincirin ikinci metilen grubu ile halka üzerindeki orto (ortho) konumundaki hidrojen atomu arasında güçlü bir sterik (steric) itme tarafından engellenir $[15,17]$.

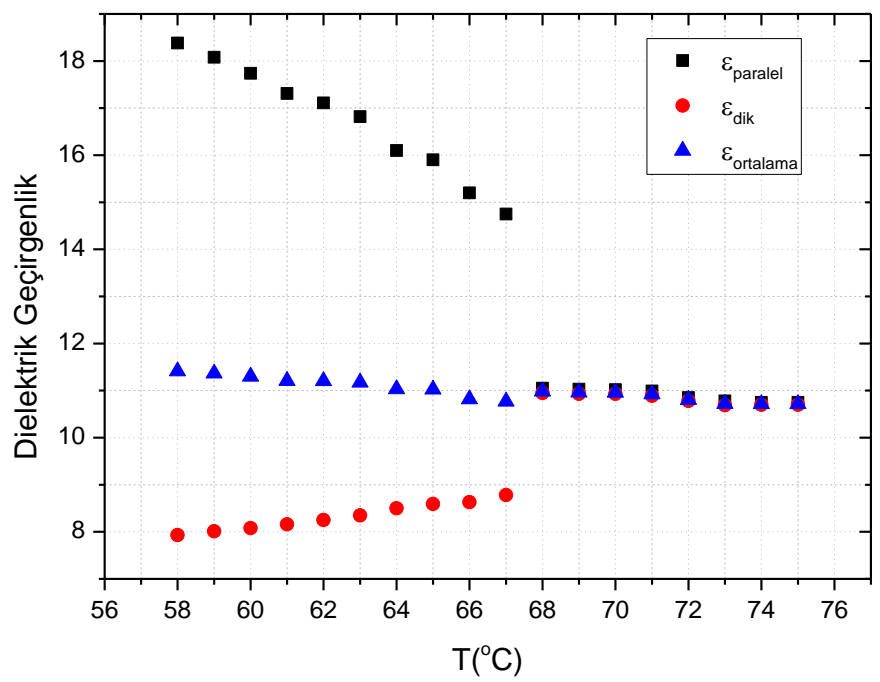

(a) 


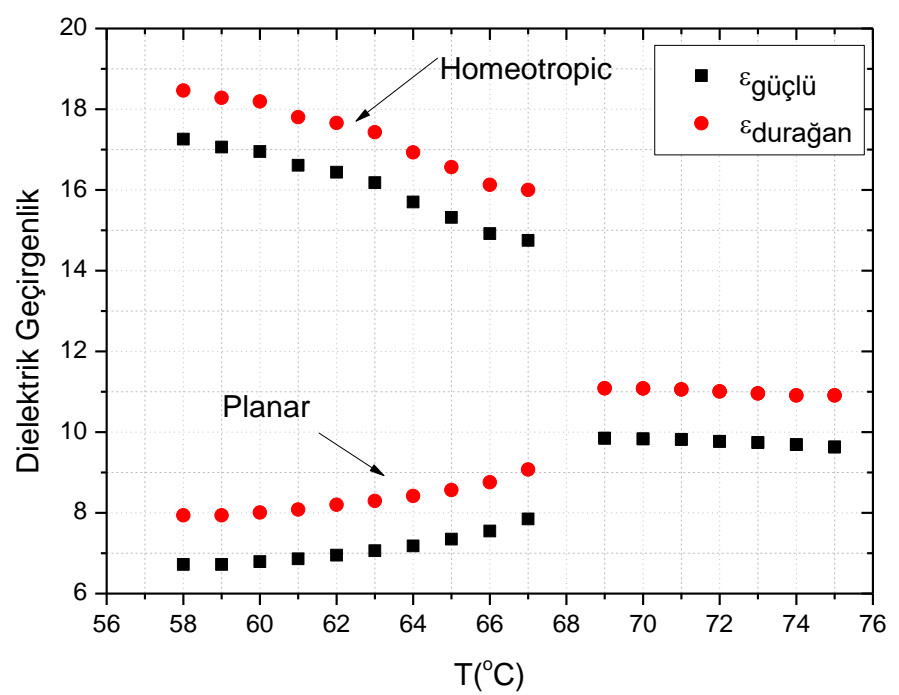

(b)

Şekil 4. (a) 4-propoxy-biphenyl-4-carbonitrile'nin dielektrik geçirgenliği (2 kHz) ve (b) izotropik ve nematik fazlardaki dielektrik gücünün sicaklığa göre değişimi.

Onsager denklemini dikkate alırsak

$\mu^{2}=g \frac{k T \varepsilon_{o}\left(\varepsilon_{S}-\varepsilon_{\infty}\right)\left(\varepsilon_{S}+\varepsilon_{\infty}\right)}{N \varepsilon_{S}\left(\varepsilon_{\infty}+2\right)}$

dipol momentin enine ve boyuna bileşenlerinin oran $1 \frac{\mu_{t}}{\mu_{l}}=\tan \phi$ şeklinde tahmin edilebilir. Bu nedenle $58^{\circ} \mathrm{C}^{\prime}$ deki $\phi$ 'nın hesaplanan değeri $35^{\circ}$ ve aynı şekilde mOCB'ler için $\mathrm{m}=5-8$ ekstrapolasyonundan $\phi$ 'nın belirlenen değerleri $32^{\circ}$ olarak belirlenmiştir [45]. Bu durum en azından izotropik fazda moleküllerin uzun ekseninin, benzen halkasının para-eksenine bu açıda eğildiğini gösterir.

Dielektrik anizotropi, dielektrik sabitinin paralel ve dik bileşenleri arasındaki farkı temsil etmektedir. Sıvı kristal molekülünün dielektrik sabiti, dipol momenti ve molekülün ana eksenine ilişkin bağıl konumu tarafından belirlenir. Bu nedenle dielektrik sabiti bir sıvı kristal malzemenin eşik voltajını etkileyen ve sıvı kristalin elektrik alanıyla etkileşim gücünü belirleyen önemli parametredir [1-5]. 4-propoxy-biphenyl-4-carbonitrile sıvı kristalinin dielektrik sabiti, nematik sıv1 kristalin yönelim derecesindeki artışı ve maddenin yoğunluğunun azalması nedeniyle sıcaklık azaldıkça artar ve tüm nematik faz boyunca dielektrik sabitinin değeri pozitiftir. Çünkü uçtaki -CN grubunun güçlü kalıcı elektrik dipol momentinin varlığı, molekülü uzun eksenine neredeyse paralel yönlendirir [28-30]. -CN grubunun benzen halkasında oluşturduğu konjügasyonu benzen halkasındaki $\pi$-elektronlarının çekilmesine izin verir ve böylelikle dipolün her iki bileşenine de belirli miktarda katkı sağlar.

\section{TARTISMA}

Bu çalışmada, 4-propoxy-biphenyl-4-carbonitrile sıvı kristalinin dielektrik özellikleri, sıcaklığın bir fonksiyonu olarak ölçülmüştür. İzotropikten nematik faza geçişte, dipollerin antiparalel olarak dizilmeleri nedeniyle sıcaklık azaldıkça ortalama dielektrik geçirgenliğin de azaldığı görülmüştür. Sözü edilen sıv1 kristal malzeme, nispeten büyük pozitif bir dielektrik anizotropiye sahiptir. Molekülün toplam dipol momentinin, molekülün uzun eksenine göre $\phi \approx 35^{\circ}$ açı ile eğimli olduğu belirlenmiştir. Dielektrik fonksiyonun sanal kısmı termal olarak uyarılmış moleküllerin uzun eksenleri etrafında takla dönüşümünü vermektedir ve gevşeme frekansı birkaç $100 \mathrm{kHz}$ mertebesindedir. Gevşeme sürecinde moleküllerin aktivasyon enerjisi Arrhenius metodu kullanılarak belirlenmiştir. Buna ek olarak elde 
edilen deneysel sonuçlar, siyanobifenil (cyanobiphenyl) bazlı sıvı kristal dimerlerin dielektrik özelliklerinin daha iyi anlaşılmasın sağlayabilecektir.

\section{KAYNAKLAR}

[1] P.G. de Gennes ve J. Prost, The Physics of Liquid Crystals, 2. Bask1, Oxford, İngiltere: Clarendon, 1993, ss. 45-57.

[2] K. Iam-Choon ve S.T. Wu, Optics and Nonlinear Optics of Liquid Crystals, 1. Bask1, Singapur: World Scientific Pub Co Inc., 1993, ss. 77-83.

[3] M. Kleman ve O.D. Lavrentovich, Soft Matter Physics: An Introduction, 1. Bask1, New York, Amerika: Springer, Verlag, 2003, ss. 127-134.

[4] S. T. Wu ve D.K. Yang, Fundamentals of Liquid Crystal Devices, 1. Bask1, West Sussex, İngiltere: Wiley, 2006, ss. 317-345.

[5] S. Singh ve D.A. Dunmur, Liquid Crystals: Fundamentals, 1. Bask1, New York, Amerika: World Scientific., 2002, ss. 102-119.

[6] P.V. Shibaev ve M.B. Guseva, "Molecular orientation in propyl-and hexyloxycyanobiphenyl vacuum deposited thin films," Journal of Crystal Growth, c. 119, s. 3-4, ss. 399-402, 1992.

[7] D. Sharma, T. Gargi, ve S.N. Tiwari, "Thermodynamical properties and infrared spectra of 4-npropoxy-4-cyanobiphenyl: Hartree-fock and density functional theory methods," Int J Electroact Mater, c. 5 , ss. 19-30, 2017.

[8] T-H. Tong, B.M. Fung, ve J.P. Bayle, "A database for the determination of orientational ordering of nine classes of liquid crystals using carbon-13 chemical shifts," Liquid crystals, c. 22, s. 2, ss. $165-169,1997$.

[9] C. David ve D. Baeyens-Volan, "Absorption and Fluorescence Spectra of 4-Cyanobiphenyl and 4'-Alkyl-or 4'-Alkoxy-Substituted Liquid Crystalline Derivatives," Molecular Crystals and Liquid Crystals, c. 59, s. 3-4, ss. 181-196, 1980.

[10] N.I. Giricheva, M.S. Fedorov, K.E. Shpilevaya, S.A. Syrbu, ve O.Y. Ditsina, "Characteristics of the hydrogen bond and the structure of H-complexes of p-n-propyloxybenzoic acid and p-npropyloxy-p'-cyanobiphenyl," Journal of Structural Chemistry, c. 58, s. 1, ss. 9-16, 2017.

[11] A.N. Isaev ve P. P. Shorygin, "Charge transfer in systems of conjugated bonds in cyanobiphenyl molecules: Quantum-chemical calculations of the structure and vibrational spectra," Russian Journal of Physical Chemistry A, c. 83, s. 3, ss. 430-435, 2009.

[12] K.I. Muta, H. Takezoe, A. Fukuda, ve E. Kuze, "Cotton-mouton effect of alkyl-and alkoxycyanobiphenyls in isotropic phase," Japanese Journal of Applied Physics, c. 18, s. 11, ss. 2073-2080, 1979.

[13] J.W. Emsley, G.R. Luckhurst, ve H.S. Sachdev, "Dependence of solute orientational order on the structure and orientational order of the liquid crystal solvent. A deuterium NMR study of p-xylened10 dissolved in a series of 4-n-alkyloxy-4'-cyanobiphenyls," Liquid Crystals, c. 5, s. 3, ss. 953-967, 1989. 
[14] F. Eikelschulte, S.Y. Yakovenko, D. Paschek, ve A. Geiger, "Electrostatic properties of cyanocontaining mesogens," Liquid Crystals, c. 27, s. 9, ss. 1137-1146, 2000.

[15] L.M. Babkov, N.I. Davydova, G.A. Puchkovskaya, ve I.N. Khakimov. "IR absorption spectra and structure of 4-cyano-4',-p-alkoxybiphenyls," Journal of Structural Chemistry, c. 34, s. 1, ss. 92-97, 1993.

[16] K.J. McEwan, S.E. Day, D.G. McDonnell, ve K.J. Harrison "Measurement of x (3) in novel liquid-crystal materials," In Nonlinear and Electro-Optic Materials for Optical Switching. International Society for Optics and Photonics, c. 1692, ss. 118-130, 1992.

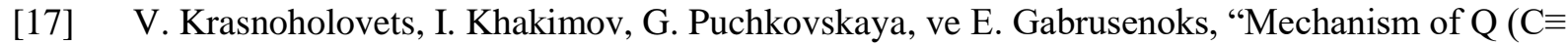
N) Band Broadening in Condensed State of Alkoxycyanobiphenyles", Molecular Crystals and Liquid Crystals Science and Technology, Section A, Molecular Crystals and Liquid Crystals, c. 348, s. 1, ss. 101-109, 2000.

[18] L.G. Kuz'mina, N.S. Kucherepa, ve A.V. Churakov, "Mesophase design: II. Molecular structure and crystal packing of 4-alkyloxycyanobiphenyls," Crystallography Reports., c. 57, s. 2, ss. 213-226, 2012.

[19] A. P. Emerson ve GR. Luckhurst, "On the relative propensities of ether and methylene linkages for liquid crystal formation in calamitics," Liquid Crystals, c. 10, s. 6, ss. 861-868, 1991.

[20] C.J. Counsell, J.W. Emsley, G.R. Luckhurst, ve HS.Sachdev, "Orientational order in the 4-nalkyloxy-4'-cyanobiphenyls: A comparison between experiment and theory," Molecular Physics, c. 63, s. 1 , ss. 33-47, 1988.

[21] L.M. Babkov, O.V. Gorshkova E.V., Gabrusyonok, G.A. Puchkovskaya, ve I.N. Khakimov, "Phase transitions, conformational lability, and intermolecular interactions in alkoxycyanobiphenyls," Journal of Structural Chemistry, c. 36, s. 5, ss. 739-746, 1995.

[22] L.M. Babkov, S.I. Tatarinov, O.V. Gorshkova, G.A. Puchkovskaya, ve I.N. Khakimov, "Polymorphism and conformational mobility of molecules of long chain aliphatic compounds," Journal of molecular structure, c. 482, ss. 453-456, 1999.

[23] L.M. Babkov, E. Gabrusenoks, V. Krasnoholovets, G.A. Puchkovskaya, ve I.N. Khakimov, "Raman spectra and molecular dynamics of alkoxycyanobiphenyles," International Society for Optics and Photonics, c. 4069, ss. 109-114, 2000.

[24] MJ. Richardson, "The derivation of thermodynamic properties by DSC: free energy curves and phase stability," Thermochimica acta, c. 229, ss. 1-4, 1993.

[25] S. Gupta ve S. Paul, "The Structure Property Relationship of a Homologous Series of Mesogenic Compounds," Molecular Crystals and Liquid Crystals Science and Technology. Section A. Molecular Crystals and Liquid Crystals, c. 260, s. 1, ss. 483-489, 1995.

[26] G.W. Gray ve A. Mosley, "Trends in the nematic-isotropic liquid transition temperatures for the homologous series of 4-n-alkoxy-and 4-n-alkyl-4'-cyanobiphenyls," Journal of the Chemical Society, Perkin Transactions, c. 2, s. 1, ss. 97-102, 1976.

[27] L.M. Babkov, E, Gabrusyonok, V.V. Krasnoholovets, G.A. Puchkovskaya, ve I.N. Khakimov, "Vibrational spectra and molecular dynamics of alkoxycyanobiphenyls," Journal of molecular structure, c. 482, ss. 475-480, 1999. 
[28] L.M. Babkov, O.V. Gorshkova, G.A. Puchkovskaya, ve I.N. Khakimov, "Vibrational spectra and structure of 4-cyano-4'-pentalkoxybiphenyl in different physical states," Journal of structural chemistry, c. 39, s. 1, ss. 44-48, 1998.

[29] V.F. Chuvaev, M.N. Rodnikova, M.R. Kiselev, A.A. Syrbu, and S.A. Syrbu, "Wide-Line 1 H NMR ve DSC study of phase states of mesomorphic states of 4'-n-alkoxy-4-biphenylcarbonitrile mesogens ( $\mathrm{n}=3$ and 7)," Russian Journal of Inorganic Chemistry, c. 54, s. 12, ss. 1969-1974, 2009.

[30] I. Penchev, I. Dozov, N. Kirov, N. Afanasyeva, ve J. Ruoliene, "Orientational Order Parameters of Some 4-Cyano 4', n-Alkoxybiphenyls," Spectroscopy Letters, c. 15, s. 4, ss. 265-274, 1982.

[31] L. M. Blinov, Structure and Properties of Liquid Crystals, 1. Bask1, New York., Amerika: Springer, 2011, ss. 19-32.

[32] L. Walz, H. Paulus, ve W. Haase, "Crystal and molecular structures of four mesogenic 4'alkoxy-4-cyanobiphenyls," Zeitschrift für Kristallographie-Crystalline Materials, c. 180, s. 1-4, ss. 97$112,1987$.

[33] J. Constant ve E.P. Raynese, "Flow aligned viscosities of cyanobiphenyls," Molecular Crystals and Liquid Crystals, c. 62, s. 1-2, ss. 115-123, 1980.

[34] J.W. Emsley, "Conformational properties of alkyloxy end chains in NOCB liquid crystals," Liquid Crystals, c. 20, ss. 1-5, 2018.

[35] J.P. Abberley, R. Killah, R. Walker, J.M. Storey, C.T. Imrie, M. Salamończyk, ve D. Pociecha, "Heliconical smectic phases formed by achiral molecules," Nat. Commun., c. 9, s. 1, ss. 1-7, 2018.

[36] D.A. Paterson, J.P. Abberley, W.T. Harrison, J. M.,Storey, ve C.T. Imrie, "Cyanobiphenylbased liquid crystal dimers and the twist-bend nematic phase," Liquid Crystals, c. 44, s. 1, ss. 127-146, 2017.

[37] M.A. Grunwald, J.C. Haenle, K.C. Kreß, R. Forschner, T. Wöhrle, W. Frey, ve S. Laschat, "Mesomorphic properties of cyanobiphenyl dimers with a central malonate unit," Liquid Crystals, c. 45, s. 11, ss. 1626-1636, 2018.

[38] D.A. Paterson, C.A. Crawford, D. Pociecha, R. Walker, J. M. Storey, E. Gorecka, ve C.T. Imrie, "The role of a terminal chain in promoting the twist-bend nematic phase: the synthesis and characterisation of the 1-(4-cyanobiphenyl-4 '-yl)-6-(4-alkyloxyanilinebenzylidene-4 '-oxy)hexanes," Liquid Crystals, c. 45, s. 13-15, ss. 2341-2351, 2018.

[39] K. Wang, M. Jirka, P. Rai, R. J. Twieg, T. Szilvási, H. Yu, ve M. Mavrikakis, "Synthesis and properties of hydroxy tail-terminated cyanobiphenyl liquid crystals," Liquid Crystals, c. 46, s. 3, ss. 397-407, 2019.

[40] W. Haase, S. Wróbel, Relaxation phenomena: liquid crystals, magnetic systems, polymers, highTc superconductors, metallic glasses, 1. Bask1, New York, Amerika: Springer Science \& Business Media, 2013, ss. 25-77.

[41] W.H. De Jeu, "Physical properties of liquid crystalline materials in relation to their applications," Molecular Crystals and Liquid Crystals, c. 63, s. 1, ss. 83-109, 1981.

[42] W. Maier ve G.A. Meier, "Simple theory of the dielectric are some homogeneous criteria oriented liquid crystal phases of nematic type," Z Naturforsch. A, c. 16, ss. 262-267, 1961. 
[43] S. Urban, B. Gestblom, D. Tuma, ve R. Dąbrowski, "Dielectric studies of two liquid crystalline benzoates with coupled and decoupled CN groups," Liquid crystals, c. 29, s. 2, ss. 301-307, 2002.

[44] P.K. Tripathi, R, Manohar, ve S.Singh, "Dielectric Relaxation Spectroscopy of Liquid Crystal in Nematogenic Mesophase," Molecular Crystals and Liquid Crystals, c. 626, s. 1, ss. 160-168, 2016.

[45] W.H. De Jeu, T.W. Lathouwers, ve P. Bordewijk, "Dielectric properties of di-n-heptyl azoxybenzene in the nematic and in the smectic-A phases," Physical Review Letters, c. 32, s. 2, ss. 4043, 1974.

[46] A. Ghanadzadeh, "Dielectric investigations and molecular association in non-mesogenic and mesogenic solutions," Journal of molecular liquids, c. 102, s. 1-3, ss. 365-377, 2003. 